\title{
Recovery of Advanced Atrioventricular Block by Cilostazol
}

\author{
Asami Nimura ${ }^{1}$, Nobuyuki Sato ${ }^{1}$, Hitoshi Sakuragi ${ }^{2}$, Satoshi Koyama ${ }^{2}$, Junichi Maruyama ${ }^{2}$, \\ Ahmed Karim Talib ${ }^{1}$, Naoki Nakagawa ${ }^{1}$, Naka Sakamoto ${ }^{1}$, Hisanobu Ota ${ }^{1}$, Yasuko Tanabe ${ }^{1}$, \\ Toshiharu Takeuchi ${ }^{1}$, Yuichiro Kawamura ${ }^{1}$ and Naoyuki Hasebe ${ }^{1}$
}

\begin{abstract}
We describe a case of advanced atrioventricular (AV) block, in which treatment with cilostazol was effective in recovering the AV conduction. The patient was referred to our hospital for close examination of the advanced AV block and permanent pacemaker implantation. Although the patient had experienced thirddegree AV block with occasional AV synchrony for more than two days, the AV conduction completely recovered after treatment with oral cilostazol at $200 \mathrm{mg}$ /day. Here we discuss the possible mechanism of the improvement in the AV conduction by cilostazol.
\end{abstract}

Key words: atrioventricular block, cilostazol

(Intern Med 50: 1957-1961, 2011)

(DOI: 10.2169/internalmedicine.50.5228)

\begin{abstract}
Introduction
Cilostazol (6[4-(1-cyclohexyl-1H-tetrazol-5-yl)butoxy]-3, 4-dihydro-2(1H)-quinolinone), a quinolinole derivative with a phoshodiesterase (PDE) type-3 inhibitory action was introduced 21 years ago in Japan and 10 years ago in the United States as an antiplatelet/antithrombic agent for patients with arterial occlusive diseases. It has been shown that cilostazol possesses a positive chronotropic effect in patients with sick sinus syndrome and in those with atrial fibrillation associated with bradycardia $(1,2)$. On the other hand, although it significantly increases the heart rate on the 24-hour Holter recordings and of the ventricular escape rate, it has been reported that cilostazol does not abolish atrioventricular abnormalities such as second-degree AV block or third-degree AV block $(2,3)$.

We encountered a case with advanced AV block in which marked sinus tachycardia with a preserved AV conduction developed after the administration of cilostazol. We hereby discuss the possible mechanism for the recovery of AV conduction brought about by cilostazol in the present case.
\end{abstract}

Case Report
An 83 -year-old woman who presented with general fa-
tigue since October 9,2010 , had suffered from hypertension,
and hence, had been treated with $5 \mathrm{mg}$ of olmesartan me-
doxomi and $0.5 \mathrm{mg}$ of indapamide in a private hospital. Al-
though her visiting nurse had pointed out that she had re-
markable bradycardia and recommended that she consult a
doctor from that time, she had refused to visit the hospital.
On October 23 , the family doctor found that her ECG ex-
hibited advanced AV block, and she was transferred to Asa-
hikawa Rehabilitation Hospital. Since she presented with ad-
vanced AV block with occasional conducted beats on admis-
sion to the hospital (Fig. 1, 2), a temporary pacemaker was
inserted from the right subclavian vein. Neither atropine sul-
fate nor beta adrenergic agents such as isoproterenol were
tried in the acute phase because the hemodynamic state of
the patient was relatively stable. No abnormalities were
found in the blood sampling or echocardiographic findings.
However, a brain MRI revealed multiple lacunar infarcts,
and remarkable coronary calcifications were observed on a
mutislice spiral CT of the heart, and hence, oral cilostazol at
200 mg was started from the second day after her admis-

${ }^{1}$ Cardiovascular Respiratory and Neurology Division, Department of Internal Medicine, Asahikawa Medical College, Japan and ${ }^{2}$ Department of Internal Medicine, Asahikawa Rehabilitation Hospital, Japan 


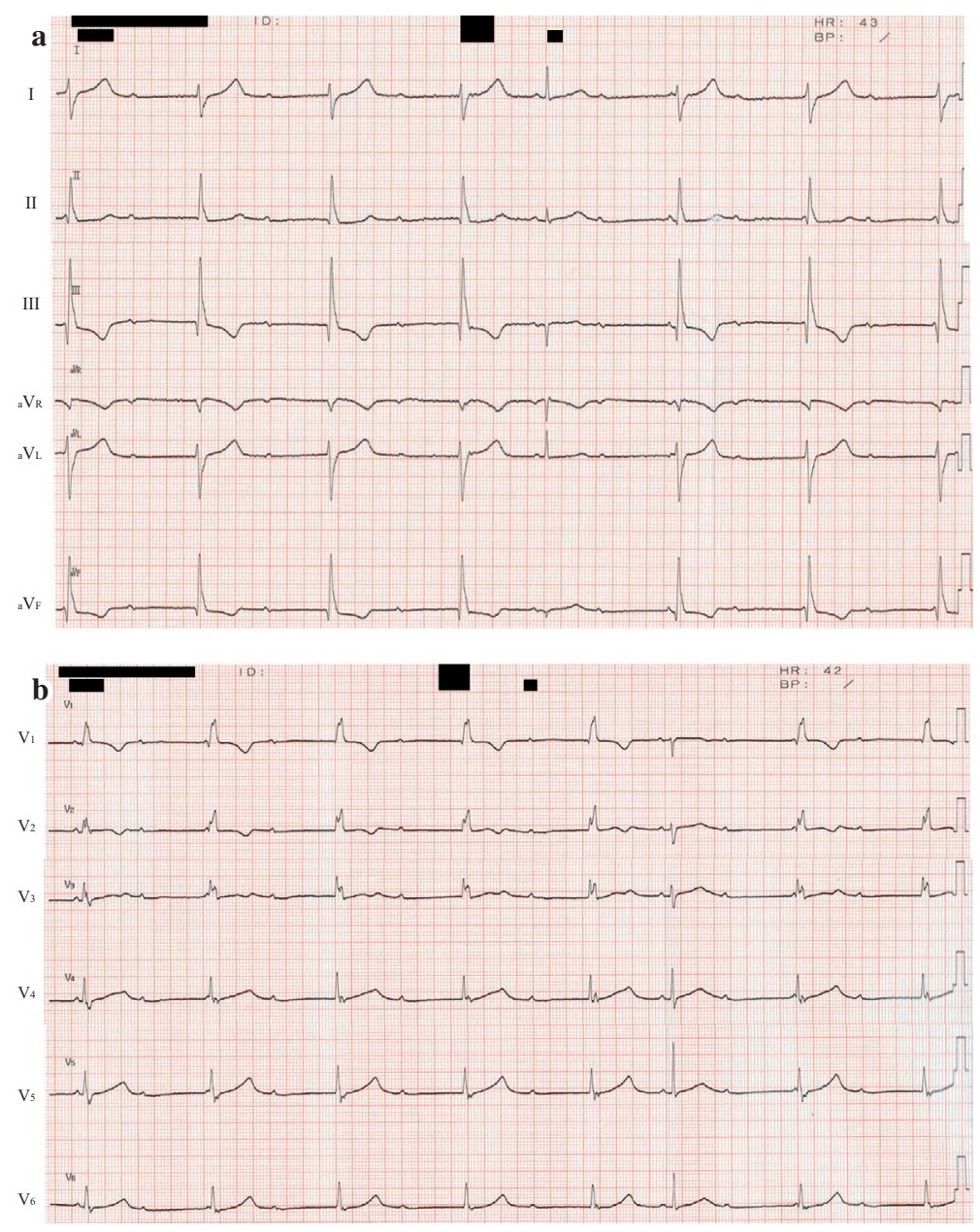

Figure 1. ECG obtained on admission at the first hospital. This ECG trace shows advanced AV block. The fifth beat in the limb leads and the sixth beat in the precordial leads were considered to be conducted beats.

sion. Surprisingly, on October 28, on the 5th day of her hospitalization, the patient's ECG exhibited sinus rhythm at a rate of $100 / \mathrm{min}$ and the temporary pacemaker was removed (Fig. 2). On November 2nd, she was admitted to the Asahikawa Medical College Hospital for close examination of the ECG abnormality and a permanent pacemaker implantation. On admission to our hospital, her ECG exhibited sinus tachycardia at a rate of 110/min (Fig. 3). Since remarkable coronary calcifications were observed on the mutislice spiral $\mathrm{CT}$, right and left cardiac catheterizations including coronary angiography were performed to rule out any ischemic heart disease or other structural heart diseases. However, no significant stenosis was found on the coronary angiogram and the cardiac catheterization data and left ventriculogram showed a normal pattern. Finally, pacemaker implantation was preformed in the patient due to the possible recurrence of advanced AV block. After one month of follow-up, the patient's ECGs have revealed normal sinus rhythm without any backup pacing.

\section{Discussion}

We described a case with advanced AV block in which cilostazol might have caused the recovery of the AV conduction. To the best of our knowledge, this is the first presentation in which cilostazol treatment might have been effective in improving advanced AV block.

First of all, we needed to rule out any structural heart disease such as ischemic heart disease or myocarditis, and secondary cardiomyopathy such as sarcoidosis. However, no abnormalities were found in the physical findings, laboratory findings, echocardiography, or cardiac catheterization including the coronary angiography and cardiac MRI, and hence, there was little possibility that any transient AV block occurred due to any apparent structural diseases as described above.

It has been demonstrated that cilostazol has positive chronotropic and dromotropic effects in patients with supraventricular bradyarrhythmias such as sick sinus syndrome or in those with Wenckebach-type atrioventricular block (1). Furthermore, previous clinical data has shown that cilostazol treatment could increase the heart rate in those with chronic atrial fibrillation associated with bradycardia (2). However, there have been few reports on the effectiveness of cilostazol in advanced AV block cases. Atarashi et al reported the chronotropic effects of cilostazol in patients with bradyar- 


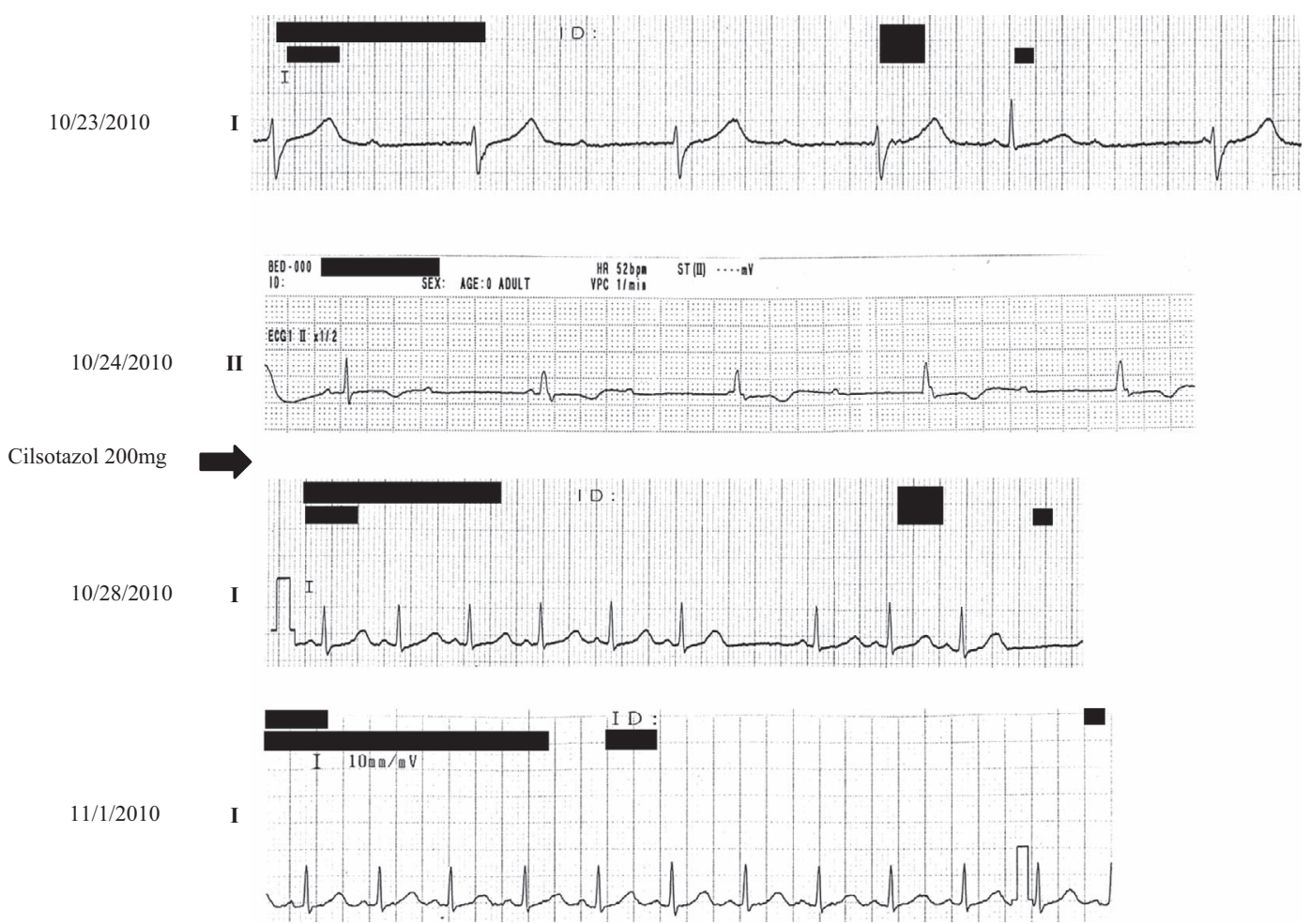

Figure 2. Time course of the ECGs. On the trace from 10/23/2010, an advanced AV block with one conducted beat (the fifth beat) was noted. On the trace from 10/24/2010, complete AV dissociation was observed. After treatment with cilostazol, a remarkable sinus tachycardia (probably associated with occasional blocked PACs) was noted.
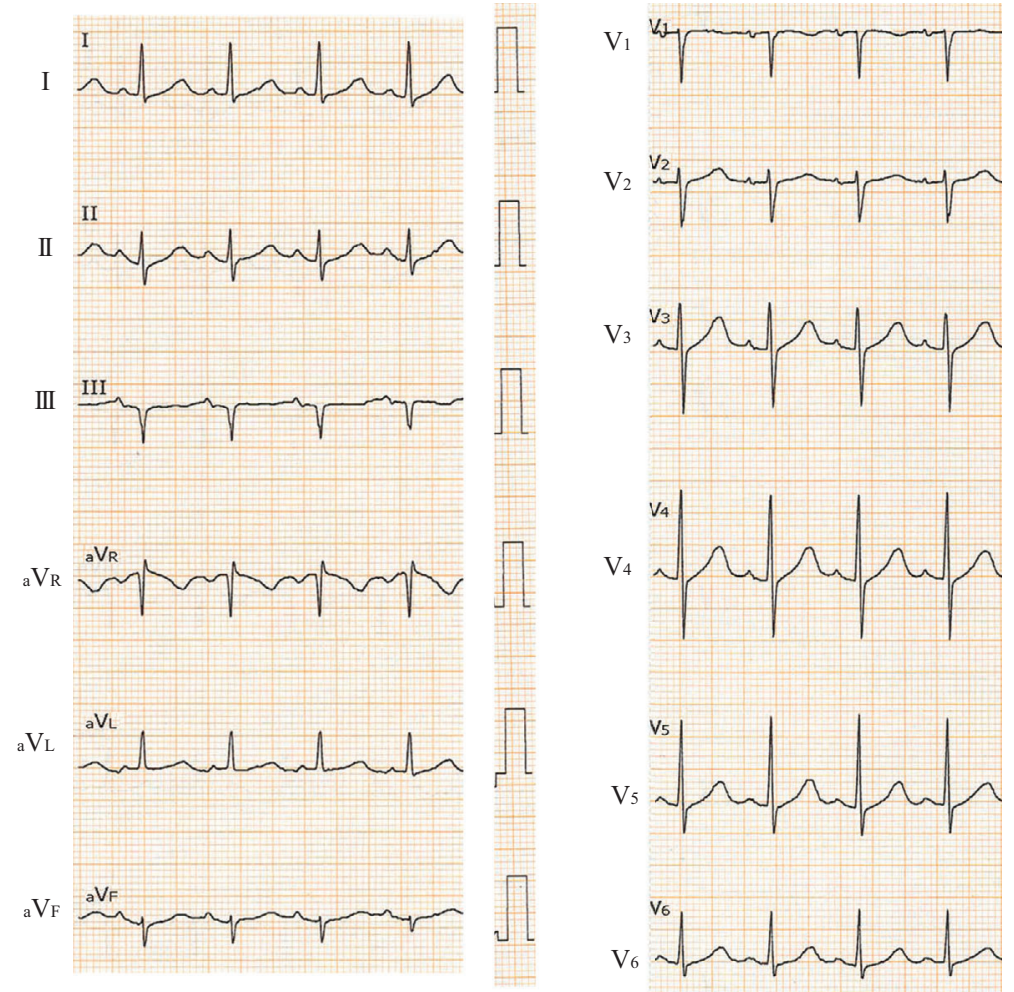

Figure 3. ECG obtained on admission at Asahikawa Medical College Hospital. This trace also shows sinus tachycardia.

rhythmias (1). According to their report, the maximum, mean, and minimum heart rates per minute after the cilosta- zol treatment increased significantly, however, Wenckebach type AV blocks were still present after the treatment in a to- 
tal of four cases with Wenckebach type AV block.

Kodama-Takahashi et al reported the effects of cilostazol on the ventricular escape rate in patients with third-degree AV block (3). According to that report, AV synchrony was seen transiently before the treatment with cilostazol in 5 of 12 study patients, and the duration of the synchrony was prolonged in three of the patients on the Holter monitoring. On the other hand, synchrony was obtained after the treatment with cilostazol in only one patient and none of the patients exhibited any abolishment of the AV block, however, it significantly increased the ventricular escape rate. Therefore, what was the mechanism for the recovery of the AV conduction in the present case? The fact that this patient had occasional AV synchrony similar to the case reported by Kodama-Takahashi et al (3), might be one of the key points for the recovery of the AV conduction. That is, occasional conducted beats, as observed in Fig. 1, 2, which would mean a partially preserved AV conduction, might be the clue to the recovery of the AV conduction. Together with recent evidence, the following possible mechanisms might be considered:

The mechanisms of cardiac pacemaking have traditionally been attributed to the actions of multiple time- and voltagedependent membrane ionic currents such as the L-type Ca current $\left(\mathrm{I}_{\mathrm{Ca}}\right)$, funny current $\left(\mathrm{I}_{\mathrm{f}}\right)$, and outward potassium current (4). Among them, the $\mathrm{I}_{\mathrm{Ca}}$ and $\mathrm{I}_{\mathrm{f}}$ are known to be controlled by intracellular cyclic AMP (cAMP) $(4,5)$. Because the AV node tissue and Purkinje fibers also express HCN4 (hyperpolarization-activated, cyclic-nucleotide gated 4) (6-8), which is responsible for the $I_{f}$ channel, it is acceptable to hypothesize that $I_{f}$ contributes to the AV node and Purkinje fibers automaticity. On the other hand, cAMP, adenyl cyclase and phoshodiesterase activity have been determined in the specialized conduction system such as the AV node, His bundle, right bundle branch, and left bundle branch of bovine hearts (9). In humans, it is also suggested that cAMP plays a key role in the sympathetic nerve/beta-adrenergic receptor/adenyl cyclase/protein kinase axis that serves to stimulate the cardiac rhythm as well as contractile force (10). Furthermore, it has also been demonstrated that PDE type-3 inhibitors accelerate the spontaneous firing rate and increase the L-type $\mathrm{Ca}^{2+}$ current in cardiac pacemaker cells (11).

In the present case, although this was within speculation, some pathological conditions such as the L-type $\mathrm{Ca}^{2+}$ channel inhibition caused by cholinergic modulation (12), antiadrenergic state caused by adenosine production (13), and disturbance of the $\mathrm{Ca}^{2+}$ handling at the sub-cellular level, might occur. In those circumstances, the PDE type-3 inhibitory activity of cilostazol might contribute to the recovery of the $\mathrm{Ca}^{2+}$ channels, $\mathrm{I}_{\mathrm{f}}$ channels, and $\mathrm{Ca}^{2+}$ handling at the subcellular level and cellular $\mathrm{Ca}^{2+}$ cycling, causing the trigger for the diastolic deporalization of the AV node tissue and Purkinje fibers. Once, the diastolic depolarization of the AV node and Purkinje fibers recovers, it is expected that the intracellular $\mathrm{Ca}^{2+}$ handling also recovers by a smooth entry of
$\mathrm{Ca}^{2+}$ via the L-type $\mathrm{Ca}^{2+}$ channels, thereby leading to a persistent recovery of the AV conduction and a good cycle of the $\mathrm{Ca}^{2+}$ handling. Of course, there is the possibility that the transient AV block in this patient occurred due to an unknown etiology (for example, previous latent myocarditis undetected by routine examinations), and the timing of the spontaneous recovery of the AV block and cilostazol treatment matched just by chance. However, as shown in Fig. 2, it was evident that the cilostazol caused a sinus tachycardia with $1: 1 \mathrm{AV}$ conduction during the development of the advanced AV block in this case. Hence, the PDE type-3 inhibitory activity of cilostazol must have played some significant role in this patient's recovery of the AV conduction.

In conclusion, it is suggested that cilostazol might be effective in advanced AV block in some particular cases due to its PDE type-3 inhibitory action and it can be a useful tool as a bridge to permanent pacemaker therapy in those cases. Since our report only describes one case, a larger population study will be needed to extract an effective group for cilostazol in AV block cases.

\section{The authors state that they have no Conflict of Interest (COI).}

\section{References}

1. Atarashi H, Endoh Y, Saitoh H, Kishida H, Hayakawa H. Chronotropic effects of cilostazole, a new antithrombotic agent, in patients with bradyarrhythmias. J Cardiovasc Pharmacol 31: 534539, 1998.

2. Toyonaga S, Nakatsu T, Murakami T, et al. Effects of cilostazol on heart rate and its variation in patients with atrial fibrillation associated with bradycardia. J Cardiovasc Pharamacol Ther 5: 183191, 2000.

3. Kodama-Takahashi K, Kurata A, Ohshima K. Effect of cilostazol on the ventricular escape rate and neurohumoral factors in patients with third-degree atrioventricular block. Chest 123: 1161-1169, 2003.

4. Di Francesco D. The role of the funny current in pacemaker activity. Circ Res 106: 434-446, 2010.

5. Irisawa H, Brown HF, Giles W. Cardiac pacemaking in the sinoatrial node. Physiol Rev 73: 197-227, 1993.

6. Hucker WJ, Nikolski VP, Efimove IR. Optimal mapping of the atrioventricular junction. J Electrocardiol 38: 121-125, 2005.

7. Han W, Bao W, Wang Z, Nattel S. Comparison of ion-channel subunit expression in canine cardiac Purkinje fibers and ventricular muscle. Circ Res 91: 790-797, 2002.

8. Baruscotti M, Barbuti A, Bucchi A. The cardiac pacemaker current. J Mol Cell Cardiol 48: 55-64, 2010.

9. Tamura Y, Saito M, Matsumura K, et al. Cyclic adenosine 3', 5'monophosphate, adenyl cyclase and phophodiesterase in the conduction system of bovine heart. Jpn Circ J 47: 351-355, 1983.

10. Fischmeister R, Castro LRV, Abi-Gerges A, et al. Compartmentation of cyclic nucleotide signaling in the heart: The role of cyclic nucleotide phosphodiesterases. Circ Res 99: 816-828, 2006.

11. Vinogradova TM, Sirenko S, Lyashkov AE, et al. Constitutive phosphodiesterase activity restricts spontaneous beating rate of cardiac pacemaker cells by suppressing local $\mathrm{Ca}^{2+}$ releases. Circ Res 102: 761-769, 2008.

12. Han X, Kobzik L, Zhao YY, et al. Nitric oxide synthase (NOS3)mediated cholinergic modulation of $\mathrm{Ca}^{2+}$ current in adult rabbit atrioventricular nodal cells. Circ Res 78: 998-1008, 1996. 
Intern Med 50: 1957-1961, 2011 DOI: 10.2169/internalmedicine.50.5228

13. van Borren MM, Verkerk AO, Wilders R, et al. Effects of muscarinic receptor stimulation on $\mathrm{Ca}^{2+}$ transient, cAMP production and pacemaker frequency of rabbit sinoatrial node cells. Basic Res Cardiol 105: 73-87, 2010.
14. Rankin AC, Martynyuk AE, Workman AJ, Kane KA. Ionic mechanisms of the effect of adenosine on single rabbit atrioventricular node myocytes. Can J Cardiol 13: 1183-1187, 1997.

(C) 2011 The Japanese Society of Internal Medicine http://www.naika.or.jp/imindex.html 